\title{
INTERACTIVE AUDIO-TEXT GUIDE FOR MUSEUM ACCESSIBILITY
}

\author{
Gavino Paddeu, Andrea Devola, Andrea Ferrero and Antonio Pintori \\ Center for Research and Scientific Studies in Sardinia, Bld. 1, Piscina Manna, Pula, Cagliari, Italy
}

\begin{abstract}
In this paper we discuss the basic architecture of a Web based information retrieval application using natural language question-answering system. The application is designed to improve the inclusiveness process. We focus on the production of audio-textual guides to make the exhibition experience accessible even by people with disabilities.
\end{abstract}

\section{KEYWORDS}

Interactive Audio Text Guide, Design for All, Museum Accessibility, Sensory Impairment, Natural Interaction, Edutainment

\section{INTRODUCTION AND STATE OF THE ART}

In this paper, we highlight the basic features to develop an inclusive Web-application for museum visitors. We have exploited the principle of universal design introduced by Dimitrova-Radojichikj, (2017) which improves the visiting experience for people with disabilities, e.g. blind and deaf people. Museums and art exhibitions are changing their look and feel: they are migrating from a traditionally based offer, which is a passive observation of art, to an immersive perception involving all the five senses. Artworks should be accessible to everyone. We found that the usual adopted mitigations, e.g. audio guides, tactile routes, guides in relief, description of artwork and information written in Braille, have obvious limitations: a traditional audio guide may not be beneficial for deaf people; some researchers have proposed specific solutions aimed at the visually impaired: Bliek, H. (2019) have shown a tactile approach by proposing a representation of a painting by 3D printing accompanied by an audio guide; Andreas Reichinger et al (October 2016) have proposed the possibility of exploring the work with the fingers, i.e. using a system based on recent low-cost depth cameras that operates directly on relief surfaces.

\section{DESCRIPTION}

The developed prototype improves the state-of-the-art by combining the use of a natural language conversational engine with an interactive tactile table to assist visually and hearing impaired people. The system interacts with the user in real-time: it is able to answer user's questions both in oral written form, while the user can explore the tactile-table (3D table in relief) representing the exposed artwork.

Our system is studied and developed for people with sensory impairments and it aims to combine the tactile experience with the sound and textual experiences in a single application, according to universal design (or design for All).

Figure 1 shows the overall of the system: 


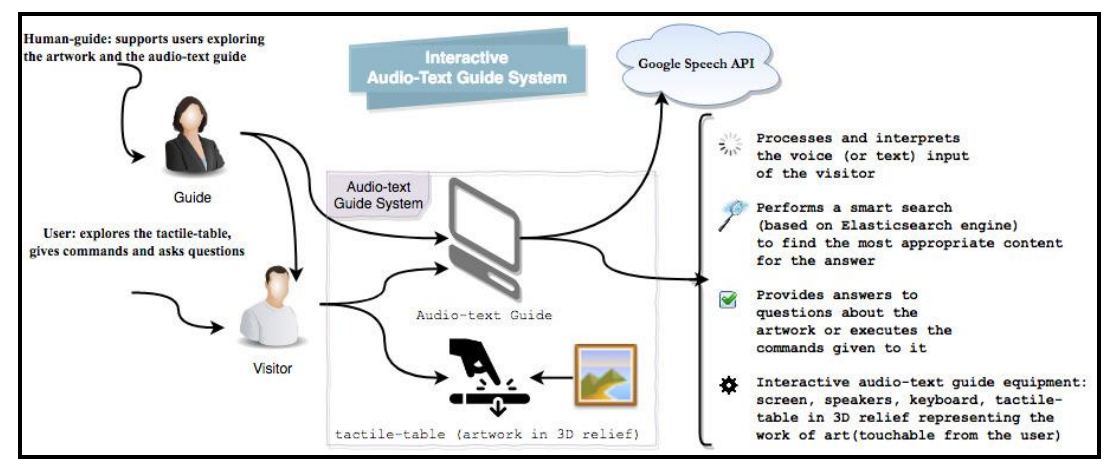

Figure 1. Audio-text guide System

The user can also explore the 3D table in relief: the system recognizes which portion is touched by means of tactile sensors inserted in the table; the audio-text guide gives a feedback customizing the content of the message based on the tactile exploration of the user, informing him or her (by voice or text) about which part of the artwork he is touching. The application supports the visually impaired (through oral exposure) as well as hearing impaired people (through the answers provided by text on screen): the user approaches the artwork with our system, equipped with video, keyboard, speakers and the tactile table. With his hands the user can touch the tactile board, which represents in 3D relief reproduction of the artwork. For the hearing impaired people the screen shows the text output of the audio-text guide and the keyboard allows the composition of questions.

The guide is also able to recognize simple oral and written commands: e.g., the current time, turn on or off the background music, or silencing the audio guide.

The Web application is developed in Javascript; it implements an audio-text guide, and it uses two main tools: the Google Speech API and Elasticsearch (it is also supported by some Node.js modules we have written).

\section{CONVERSATIONAL ENGINE AND INTERACTIVE TACTILE-TABLE 3D}

For the realization of our audio-text guide we have thought of a prototype of a conversational engine, resulting from the studies in the NLP field and related to Speech to Text and Text to Speech topics. It exploits the potential of Elasticsearch, with full-text capability, which has been specifically configured so that it can easily create a match between the question asked by the user and the contents of a database.

We used ElasticSearch queries, as shown in Figure 2. The list of questions (related to the artwork) and possible answers provided has been stored in JSON format. The adopted JSON format distinguishes between mandatory information that must be presented to the user and optional information that might be presented to the user. Moreover, with the appropriate use of the boost option it is possible to assign different weights to different parts of the text.

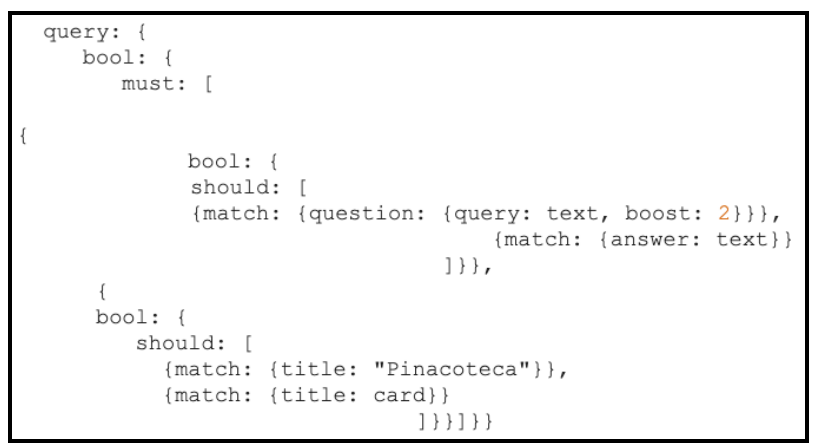

Figure 2. ElasticSearch query 
As already mentioned, the prototype includes an interactive tactile 3D table (containing the artwork in relief) that recognizes which part of it is touched and then informs the user via audio/textual feedback.

The interactive touch table was produced in three main stages: 1) Image acquisition and processing of the artwork. 2) Transformation of the 2D image into a 3D representation and simplification of the details (keeping the peculiar characteristics indicated by art experts). 3) Creation of the tactile table using a 3D printer. Figure 3 shows an example of such a table and its processing:

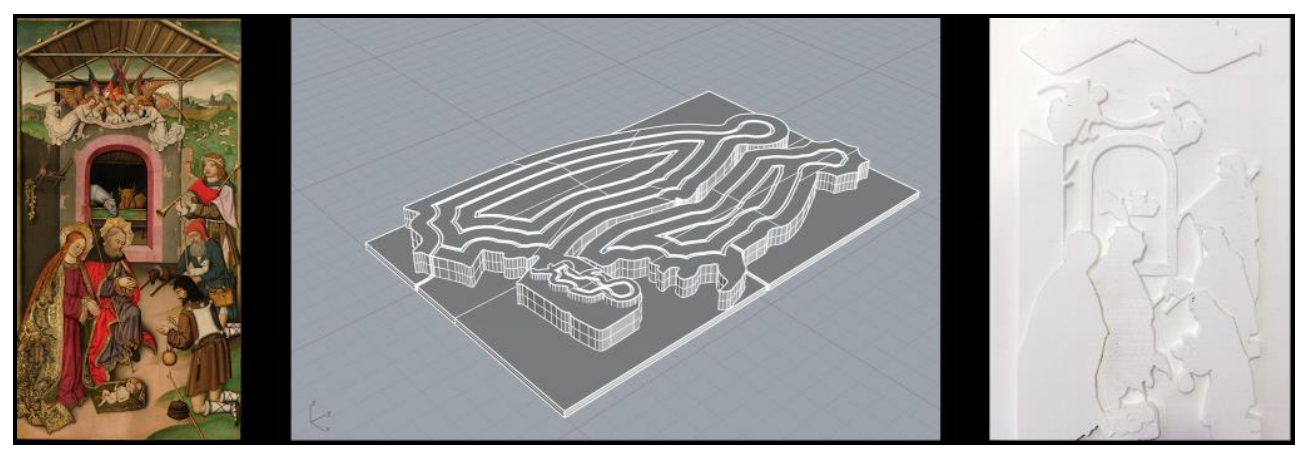

Figure 3. The artwork "Adorazione dei Pastori”; transformation into 3D representation; 3D printed table

\section{TEST AND CONCLUSION}

The prototype, being developed in collaboration with local and national museums and people with sensory disabilities, has been tested by fifteen people and partners of the project, by blind and able-bodied.

Each participant asked about twenty questions to the audio-text guide and we recorded every result. First assessment gave a positive feedback showing $80 \%$ overall satisfaction.

We have planned a complete test to measure the benefits achieved with this work with a larger group of people.

The prototype will be available for testing during the conference, along with the poster for this article.

\section{ACKNOWLEDGEMENT}

This work is part of the Overtheview project, funded by POR FESR 2014/2020 funds, Priority Axis I "Scientific Research, Technological Development and Innovation".

Thanks to the "Polo Museale della Sardegna" and the "Pinacoteca Nazionale di Cagliari" for collaboration.

\section{REFERENCES}

Bliek, H. et al, 2019. Making art accessible to visually impaired museum visitors. Delft University of Technology, Delft, Netherlands.

Dimitrova-Radojichikj, D., 2017. Museums: Accessibility to visitors with visual impairment. Skopje, Republic of Macedonia, p. 2.

Elasticsearch, (https://www.elastic.co/).

Reichinger, A. et al, 2016. A Concept for Re-useable Interactive Tactile Reliefs. Wien, Austria. 